\title{
Limit behaviour of the minimal solution of a BSDE with singular terminal condition in the non Markovian setting
}

\author{
Dmytro Marushkevych • Alexandre Popier iD
}

Received: 8 March 2019 / Accepted: 10 January 2020 / Published online: 19 February 2020 (c) The Author(s). 2020 Open Access This article is distributed under the terms of the Creative Commons Attribution 4.0 International License (http://creativecommons.org/licenses/by/4.0/), which permits unrestricted use, distribution, and reproduction in any medium, provided you give appropriate credit to the original author(s) and the source, provide a link to the Creative Commons license, and indicate if changes were made.

Abstract We use the functional Itô calculus to prove that the solution of a BSDE with singular terminal condition verifies at the terminal time: $\liminf _{t \rightarrow T} Y(t)=\xi=$ $Y(T)$. Hence, we extend known results for a non-Markovian terminal condition.

Keywords Backward stochastic differential equations · Functional stochastic calculus · Singularity

AMS class $60 \mathrm{G} 99 \cdot 60 \mathrm{H} 99$

\section{Introduction}

In this paper, we consider a filtered probability space $(\Omega, \mathcal{F}, \mathbb{F}, \mathbb{P})$ with a complete and right-continuous filtration $\mathbb{F}=\left\{\mathcal{F}_{t}, t \geq 0\right\}$. We assume that this space supports a Brownian motion $W$. We consider the following BSDE:

$$
Y(t)=\xi+\int_{t}^{T} f(s, Y(s), Z(s)) d s-\int_{t}^{T} Z(s) d W(s)-\int_{t}^{T} d M(s),
$$

where $f$ is the generator and $\xi$ is the terminal condition. The solution is the triplet $(Y, Z, M)$. Since no particular assumption is made on the underlying filtration, there is the additional martingale part $M$ orthogonal to $W$. It is already established that such a BSDE has a unique solution when the terminal condition $\xi$ belongs to

D. Marushkevych and A. Popier $(\square)$

Laboratoire Manceau de Mathématiques, Le Mans Université, Avenue Olivier Messiaen, 72085 Le

Mans cedex 9, France

e-mail: Alexandre.Popier@univ-lemans.fr

D. Marushkevych

e-mail: Dmytro.Marushkevych.Etu@univ-lemans.fr 
$L^{p}\left(\Omega, \mathcal{F}_{T}, \mathbb{P}\right), p>1$, when $f$ satisfies some regularity assumptions w.r.t. $y$ and $z$ and when the process $f(t, 0,0)$ is in $L^{p}([0, T] \times \Omega)$ (see, among others, (Delong 2013), (Kruse and Popier 2016a), or (Pardoux and Rascanu 2014)).

When the terminal condition $\xi$ satisfies

$$
\mathbb{P}(\xi=+\infty)>0,
$$

we call the BSDE singular. This singular case has been studied in Popier (2006) when the filtration is generated by the Brownian motion (no additional noise, i.e., $M=0$ ) and for the particular generator $f(t, y, z)=f(y)=-y|y|^{q}, q>0$. Extensions have been studied in Kruse and Popier (2016b) and Popier (2016). Recently, singular BSDEs were used to solve a particular stochastic control problem with application to portfolio management (see Ankirchner et al. (2014), Graewe et al. (2015), Kruse and Popier (2016b)). In this framework, the generator does not depend on $z$ and has the following form

$$
f(t, y, z)=-\frac{y|y|^{q}}{q \alpha(t)^{q}}+\gamma(t)
$$

where $\alpha$ and $\gamma$ are positive processes. The minimal solution $(Y, Z, M)$ (provided it exists) gives the value function of the following control problem: minimize ${ }^{1}$

$$
\mathbb{E}\left[\int_{t}^{T}\left(\alpha(s)|\eta(s)|^{p}+\gamma(s)|X(s)|^{p}\right) d s+\xi|X(T)|^{p} \mid \mathcal{F}_{t}\right]
$$

over all progressively measurable processes $X$ that satisfy the dynamics

$$
X(s)=x+\int_{t}^{s} \eta(u) d u
$$

and the terminal state constraint

$$
X(T) \mathbf{1}_{\xi=\infty}=0 .
$$

In (4), the constant $p$ is the Hölder conjugate of $1+q$. For the financial point of view, the set $\{\xi=+\infty\}$ is a specification of a set of market scenarios where liquidation is mandatory. The value function is equal to $|x|^{p} Y(t)$ and the optimal state process $X^{*}$ can be computed directly with $Y$. Note that the martingale part of the solution $(Z, M)$ is not employed in the computation of the optimal state process. Thus, the control problem can be completely solved provided the BSDE has a minimal solution (see section 2 and Theorem 4 in Kruse and Popier (2016b) for more details on the control problem).

In Kruse and Popier (2016b), it is proved that under Conditions (A) on $f$ (see Section 2), the BSDE (1) with singular terminal condition (2) has a minimal supersolution $(Y, Z, M)$ such that a.s.

$$
\liminf _{t \rightarrow T} Y(t) \geq \xi
$$

The main requirement is that $f$ decreases w.r.t. $y$ at least as a polynomial function (

\footnotetext{
${ }^{1}$ with the convention $0 . \infty=0$.
} 
a priori estimate, which states that $Y(t)$ is bounded from above for any $t<T$ by a finite process (Inequality (9)).

In the classical setting $\left(\xi \in L^{p}(\Omega)\right), Y$ has a limit as $t$ increases to $T$ since the process is a solution of the BSDE (1) and thus is càdlàg ${ }^{2}$. Moreover this limit is equal to $\xi$ a.s. if the filtration $\mathbb{F}$ is left-continuous at time $T$. Indeed, for a complete and right-continuous filtration, the orthogonal martingale $M$ may have a jump at time $T$; for example, if $T$ is a thin time. In this case:

$$
\lim _{t \rightarrow T} Y(t)=\xi-\Delta M(T)=Y(T)-\Delta M(T)
$$

(see the discussion in (Popier 2016, section 2.2)). Left-continuity at time $T$ of the filtration $\mathbb{F}$ is a sufficient condition to avoid this issue (and to obtain the behaviour (5) of the super-solution $Y$ at time $T$ ). For example, the filtration $\mathbb{F}$ generated by the Brownian motion $W$ is left-continuous at time $T$ (there is no additional martingale $M$ under this setting).

For the related control problem (4), this weak behaviour (5) at time $T$ of the minimal process $Y$ is sufficient to obtain the optimal control and the value function (see Kruse and Popier (2016b)). Nevertheless, two natural questions arise here:

1. Does the limit exist ?

2. Can the inequality (5) be an equality if the filtration is left-continuous at time $T$ ?

This paper addresses the second question. Despite the very theoretical aspect of these questions, there are several applications. From a financial point of view, it means that the optimal liquidation portfolio does not super hedge the penalty cost $\xi$. And in Bank and Voß (2018), a positive answer to these questions is a condition for solving the optimal targeting problem.

\subsection{Related literature}

To our best knowledge, there are only three works on this topic: (Popier 2006), (Popier 2016), and (Sezer et al. 2019). In Popier (2006), the author works under the Brownian setting, that is, when the filtration $\mathbb{F}$ is generated by the Brownian motion $W$, and with the generator $f(t, y, z)=-y|y|^{q}$. He proved that the limit of $Y$ at time $T$ always exists (see (Popier 2006, Proposition 9)), and to obtain the equality:

$$
\lim _{t \rightarrow T} Y(t)=\xi
$$

it is supposed that $\xi=g(X(T))$, where $X$ is the solution of a forward SDE. Two cases are distinguished:

- When $q>2$ without additional conditions since there exists a suitable control of $Z$.

- When $q \leq 2$ but with Malliavin calculus: roughly speaking, $Z$ is the Malliavin derivative of $Y$ and the author uses an integration by parts to remove $Z$.

\footnotetext{
${ }^{2}$ French acronym for right continuous with left limits.
} 
In Popier (2016), the author deals with a generator satisfying Condition (A) (see below). The filtration $\mathbb{F}$ should be left-continuous at time $T$.

1 The existence of a limit at time $T$ is proved under a structural condition on the generator $f$ (Popier 2016, Theorem 3.1). Roughly speaking, it is proved that $Y$ is a non linear continuous transform of a nonnegative supermartingale. Relaxing the condition on $f$ is not the aim of this paper.

2. In Popier (2016), there is also an extension of the result concerning the second question, again, when $\xi=g(X(T))$. This setting is called half-Markovian since a similar condition on $f$ is not required. We only suppose that for any $(y, z)$, $t \mapsto f(t, y, z)$ is progressively measurable.

The paper (Sezer et al. 2019) was the first attempt to prove the equality when $\xi$ is not given by $g(X(T))$ in the Brownian setting. Indeed, $\xi$ was assumed to be equal to $\infty \mathbf{1}_{B(m, r)^{c}}\left(W_{T}\right)$ or $\infty \mathbf{1}_{B(m, r)}\left(W_{T}\right)$, where $W_{T}$ denotes the whole trajectory $(W(t), t \in[0, T])$ and $B(m, r)$ is the ball in the space $C([0, T])$ centered at the constant function $m$ and radius $r$. The proof was based on the exit time of the Brownian motion and the derivation and solution of a related heat equation with a singular and discontinuous Dirichlet boundary condition. Let us remark that the considered functionals are not continuous, in the sense of (Cont and Fournie 2013, Definition $2.3)$.

\subsection{Contributions and composition of the paper}

Our goal is to give another class of non-Markovian terminal values $\xi$ such that the minimal supersolution constructed in Kruse and Popier (2016b) satisfies:

$$
\liminf _{t \rightarrow T} Y(t)=\xi
$$

This class is constructed using the functional Itô calculus developed by Cont (2016); Cont and Fournié (2010); Cont and Fournié (2013); Dupire (2009) (see Cont (2016) for an overview and the references therein). Roughly speaking, $\xi$ is a smooth functional $F$ of the paths of a continuous diffusion process $X$ (solution of the SDE (14)) and of its bracket $[X]$, satisfying some integrability assumption (see Condition (C)). As presented in subsection 3.2, our condition includes the Markovian case studied in Popier (2016), but also the integral of $X$ w.r.t. $t$ or some approximation of the process $X$.

The paper is organized as follows. In Section 2, we recall known results concerning BSDEs with a singular terminal condition and the functional Itô calculus, in particular the definition of smooth functionals. In Section 3, we give the setting of our continuity result (Condition $(\mathbf{C})$ ) and we state our result (Theorem 1). In the rest of the section we prove our statement and provide several examples satisfying our required assumptions.

To finish this introduction, let us discuss some points, which are left as future research. From Cont (2016), we know that the functional Itô calculus is also valid for more general semimartingales $X$. In particular continuity is not really relevant in the framework of Cont (2016). However, the presence of jumps requires a very careful 
discussion about the possibility of jumping inside the singularity set of $\xi$ (see Popier (2016) for the Markovian case). This is the reason why we impose the continuity of $X$. Moreover to avoid very technical arguments, we do not consider locally smooth functionals (see (Cont 2016, Definition 5.2.10)).

\section{Setting and known results}

We consider a filtered probability space $\left(\Omega, \mathcal{F}, \mathbb{P}, \mathbb{F}=\left(\mathcal{F}_{t}\right)_{t \geq 0}\right)$. The filtration is assumed to be complete and right-continuous. Note that all martingales have rightcontinuous modifications in this setting and we will always assume that we are taking the right continuous version. We assume that $\left(\Omega, \mathcal{F}, \mathbb{P}, \mathbb{F}=\left(\mathcal{F}_{t}\right)_{t \geq 0}\right)$ supports a $d$-dimensional Brownian motion $W$. In this paper, for a given $T \geq 0$, we denote:

- $\quad \mathcal{D}\left(\right.$ resp., $\mathcal{D}(0, T)$ ): the set of all predictable processes on $\mathbb{R}_{+}$(resp., on $\left.[0, T]\right)$. $L_{\text {loc }}^{2}(W)$ is the subspace of $\mathcal{D}$ such that for any $t \geq 0$ a.s.

$$
\int_{0}^{t}|Z(s)|^{2} d s<+\infty .
$$

- $\mathcal{M}_{\text {loc }}$ : the set of càdlàg local martingales orthogonal to $W$. If $M \in \mathcal{M}_{l o c}$ then,

$$
\left[M, W^{i}\right](t)=0,1 \leq i \leq k
$$

- $\mathcal{M}$ is the subspace of $\mathcal{M}_{\text {loc }}$ of martingales.

On $\mathbb{R}^{d},|$.$| denotes the Euclidean norm and \mathbb{R}^{d \times d^{\prime}}$ is identified with the space of real matrices with $d$ rows and $d^{\prime}$ columns. If $z \in \mathbb{R}^{d \times d^{\prime}}$, we have $|z|^{2}=\operatorname{trace}\left(z z^{*}\right)$.

Now, to define the solution of our BSDE, let us introduce the following spaces for $p \geq 1$.

- $\quad \mathbb{D}^{p}(0, T)$ is the space of all adapted càdlàg processes $X$ such that

$$
\mathbb{E}\left(\sup _{t \in[0, T]}|X(t)|^{p}\right)<+\infty .
$$

For simplicity, $X_{*}=\sup _{t \in[0, T]}|X(t)|$.

- $\quad \mathbb{H}^{p}(0, T)$ is the subspace of all processes $X \in \mathcal{D}(0, T)$ such that

$$
\mathbb{E}\left[\left(\int_{0}^{T}|X(t)|^{2} d t\right)^{\frac{p}{2}}\right]<+\infty .
$$

- $\quad \mathbb{M}^{p}(0, T)$ is the subspace of $\mathcal{M}$ of all martingales such that

$$
\mathbb{E}\left[([M](T))^{\frac{p}{2}}\right]<+\infty .
$$

- $\quad \mathbb{S}^{p}(0, T)=\mathbb{D}^{p}(0, T) \times \mathbb{H}^{p}(0, T) \times \mathbb{M}^{p}(0, T)$. 
If $M$ is an $\mathbb{R}^{d}$-valued martingale in $\mathcal{M}$, the bracket process $[M](t)$ is

$$
[M](t)=\sum_{i=1}^{d}\left[M^{i}\right](t),
$$

where $M^{i}$ is the $i$-th component of the vector $M$.

We consider the BSDE (1)

$$
Y(t)=\xi+\int_{t}^{T} f(s, Y(s), Z(s)) d s-\int_{t}^{T} Z(s) d W(s)-\int_{t}^{T} d M(s) .
$$

Here, the random variable $\xi$ is $\mathcal{F}_{T}$-measurable with values in $\mathbb{R}$ and the generator $f: \Omega \times[0, T] \times \mathbb{R} \times \mathbb{R}^{d} \rightarrow \mathbb{R}$ is a random function, measurable with respect to Prog $\times \mathcal{B}(\mathbb{R}) \times \mathcal{B}\left(\mathbb{R}^{d}\right)$ where Prog denotes the sigma-field of progressive subsets of $\Omega \times[0, T]$. The unknowns are $(Y, Z, M)$ such that

- $\quad Y$ is progressively measurable and càdlàg with values in $\mathbb{R}$;

- $Z \in L_{l o c}^{2}(W)$, with values in $\mathbb{R}^{d}$;

- $M \in \mathcal{M}_{\text {loc }}$ with values in $\mathbb{R}$.

For notational convenience, we denote $f^{0}(t)=f(t, 0,0)$.

\subsection{Assumptions}

(A1) $\xi$ and $f^{0}$ are nonnegative and $\mathbb{P}(\xi=+\infty)>0$. $\mathfrak{S}_{\xi}$ is the $\mathcal{F}_{T}$-measurable set of singularity:

$$
\mathfrak{S}_{\xi}=\{\xi=+\infty\}
$$

(A2) The function $y \mapsto f(t, y, z)$ is continuous and monotone: there exists $\chi \in \mathbb{R}$ such that a.s. and for any $t \in[0, T]$ and $z \in \mathbb{R}^{k}$

$$
\left(f(t, y, z)-f\left(t, y^{\prime}, z\right)\right)\left(y-y^{\prime}\right) \leq \chi\left(y-y^{\prime}\right)^{2} .
$$

(A3) For every $n>0$, the function

$$
\sup _{|y| \leq n}\left|f(t, y, 0)-f_{t}^{0}\right| \in L^{1}((0, T) \times \Omega) .
$$

(A4) $f$ is Lipschitz in $z$, uniformly w.r.t. all parameters: there exists $L>0$ such that for any $(t, y), z$ and $z^{\prime}:$ a.s.

$$
\left|f(t, y, z)-f\left(t, y, z^{\prime}\right)\right| \leq L\left|z-z^{\prime}\right| .
$$

Note that no assumption on $f^{0}$ (except nonnegativity) is required. Conditions (A1)-(A4) will ensure existence and uniqueness of the solution for a version of BSDE (1), where the terminal condition $\xi$ is replaced by $\xi \wedge n$ and where the generator $f$ is replaced by $f_{n}=f-f^{0}+\left(f^{0} \wedge n\right)$ for some $n>0$ (see BSDE (7) below). We obtain the minimal supersolution (see Proposition 1) with singular terminal condition $\xi$ by letting the truncation $n$ tend to $\infty$. To ensure that in the limit (when $n$ goes 
to $\infty$ ) the solution component $Y$ attains the value $\infty$ on $\mathfrak{S}_{\xi}$ at time $T$ but is finite before time $T$, we suppose that:

(A5) There exists a constant $q>0$ and a positive process $a$ such that for any $y \geq 0$

$$
f(t, y, z) \leq-(a(t)) y|y|^{q}+f(t, 0, z) .
$$

Moreover, in order to derive the a priori estimate, the following assumption is made.

(A6) There exists some $\ell>1$ such that

$$
\mathbb{E} \int_{0}^{T}\left[\left(\frac{1}{q a(s)}\right)^{\frac{\ell}{q}}+\left(f^{0}(s)\right)^{\ell}\right] d s<+\infty
$$

Definition 1 The generator $f$ satisfies Condition (A) if all assumptions (A1)-(A6) hold.

Example 1 (Toy example) The function $f(y)=-y|y|^{q}$ satisfies all previous conditions. It corresponds to generator (3) with $\alpha(t)=(1 / q)^{1 / q}$ and $\gamma(t)=0$.

Example 2 (Portfolio example) The generator $f$ given by (3) verifies the required assumptions with $a(t)=1 /(q \alpha(t))^{q}$ if $\mathbb{E} \int_{0}^{T}\left(\alpha(s)^{\ell}+\gamma(s)^{\ell}\right) d s<+\infty$.

Remarks 1 In Kruse and Popier (2016b) or in Popier (2016), we consider some weaker integrability conditions on $f^{0}$ (see (A6) in Kruse and Popier (2016b) and $\left(A 6^{*}\right)$ and (A8) in Popier (2016). These weak hypotheses are also assumed here. But since it is not the core of this paper, we work under the stronger (but easier to check) Condition 2.1 on $f^{0}$.

\subsection{Known results}

In Kruse and Popier (2016a; 2017), we proved that if $\xi \in L^{p}(\Omega)$, for some $p>1$, then under Conditions (A) there exists a unique solution $(Y, Z, M)$ in $\mathbb{S}^{p \wedge \ell}(0, T)$ to BSDE (1). Since we assume completeness and right-continuity of the filtration $\mathbb{F}$, the process $Y$ has the same regularity as the martingale $M$, namely, it is càdlàg. In particular, the limit at time $T$ of $Y$ exists.

As mentioned before, a way to construct the minimal supersolution (Ankirchner et al. 2014; Kruse and Popier 2016b; Popier 2006) is to approximate our BSDE by considering a terminal condition of the form $\xi^{n}:=\xi \wedge n$ and observe asymptotic behaviour. In the rest of the paper, $\left(Y^{n}, Z^{n}, M^{n}\right)$ will be the solution of the truncated BSDE:

$$
Y^{n}(t)=\xi \wedge n+\int_{t}^{T} f_{n}\left(s, Y^{n}(s), Z^{n}(s)\right) d s-\int_{t}^{T} Z^{n}(s) d W(s)-\int_{t}^{T} d M^{n}(s) .
$$


Here, $f_{n}(t, y, z)$ is the generator obtained by the truncation on $f^{0}$ :

$$
f_{n}(t, y, z)=\left(f(t, y, z)-f^{0}(t)\right)+\left(f^{0}(t) \wedge n\right) .
$$

Under (A1)-(A4), existence and uniqueness of $\left(Y^{n}, Z^{n}, M^{n}\right)$ comes from Kruse and Popier (2016a, Theorem 2). Moreover, using a comparison argument (see Kruse and Popier (2016a) or Quenez and Sulem (2013)) we obtain for $m \leq n$ : $0 \leq Y^{m}(t) \leq$ $Y^{n}(t)$. This allows us to define $Y$ as the limit of the increasing sequence $\left(Y_{t}^{n}\right)_{n \geq 1}$ :

$$
\forall t \in[0, T], \quad Y(t):=\lim _{n \rightarrow \infty} Y^{n}(t) .
$$

Using (A5) and (A6), the key point in the construction of the solution is the following a priori estimate (Kruse and Popier 2016b, Proposition 2): for any $n$

$$
\begin{aligned}
Y^{n}(t) & \leq \frac{K_{\ell, L}}{(T-t)^{1+1 / q}}\left\{\mathbb{E}\left(\int_{t}^{T}\left[\left(\frac{1}{q a(s)}\right)^{\frac{1}{q}}+(T-s)^{1+1 / q} f^{0}(s)\right]^{\ell} d s \mid \mathcal{F}_{t}\right)\right\}^{\frac{1}{\ell}} \\
& =\frac{K_{\ell, L}}{(T-t)^{1+1 / q}} \Gamma(t),
\end{aligned}
$$

where $K_{\ell, L}$ is a nonnegative constant depending only on $\ell$ and $L$ and this constant is a nondecreasing function of $L$ and a nonincreasing function of $\ell$. Condition 2.1 implies that a.s. $Y(t)<+\infty$ on $[0, T)$.

\section{Remarks 2}

- The constants $K_{\ell, L}$ and $\ell>1$ come from the growth condition on $f$ w.r.t. $z$. In other words, iff does not depend on $z$, we take $K_{\ell, L}=\ell=1$.

- If $f(y)=-y|y|^{q}, a(t)=1, L=0$, and (9) is equal to the estimate of Popier (2006), :

$$
Y_{t} \leq\left(\frac{1}{q(T-t)}\right)^{1 / q}
$$

By the stability of the solutions of BSDEs (Kruse and Popier 2016b, Proposition 3 ), there exists a constant $C$ such that for any $0<t<T$ and all $n$ and $m$

$$
\begin{array}{r}
\mathbb{E}\left[\sup _{0 \leq s \leq t}\left|Y^{n}(s)-Y^{m}(s)\right|^{\ell}+\left(\int_{0}^{t}\left|Z^{n}(s)-Z^{m}(s)\right|^{2} d s\right)^{\ell / 2}+\left[M^{n}-M^{m}\right](t)^{\ell / 2}\right] \\
\leq C \mathbb{E}\left[\left|Y^{n}(t)-Y^{m}(t)\right|^{\ell}\right]+C \mathbb{E} \int_{0}^{t}\left|f^{0}(s) \wedge n-f^{0}(s) \wedge m\right|^{\ell} d s .
\end{array}
$$

Since $Y^{n}(t)$ converges to $Y(t)$ almost surely, with the a priori estimate (9), Condition 2.1, and the dominated convergence theorem, we deduce that for every $\varepsilon>0$, $\left(Y^{n}, Z^{n}, M^{n}\right)_{n \geq 1}$ converges to $(Y, Z, M)$ in $\mathbb{S}^{\ell}(0, T-\varepsilon)$. Then passing to the limit in (7), we get for all $0 \leq s \leq t<T$ :

$$
Y(s)=Y(t)+\int_{s}^{t} f(t, Y(r), Z(r)) d r-\int_{s}^{t} Z(r) d W(r)+M(t)-M(s) .
$$


Note that all of these results are obtained without the left-continuity assumption on the filtration $\mathbb{F}$.

Since the solution $(Y, Z, M)$ satisfies the preceding dynamic (11) only on $[0, T-$ $\varepsilon]$ for any $\varepsilon>0$, we cannot directly derive existence of the left limit at time $T$ for $Y$. However, if for any $n$

$$
\lim _{t \rightarrow T} Y^{n}(t)=\xi \wedge n,
$$

we deduce (5), i.e. lower semi-continuity of $Y$ at time $T$. Left-continuity of the filtration $\mathbb{F}$ at time $T$ is sufficient to get this equality on $Y^{n}$.

Finally if $(\tilde{Y}, \tilde{Z}, \tilde{M})$ belongs to $\mathbb{S}^{\ell}(0, t)$ for any $t<T$ and fixed $\ell>1$ and if $\tilde{Y} \geq 0$ and satisfies (11) and (5), then by the comparison principle between $\widetilde{Y}$ and $Y^{n}$, we obtain that a.s. for any $t \in[0, T], \widetilde{Y}_{t} \geq Y_{t}^{n}$. The properties of $(Y, Z, M)$ are gathered in:

Proposition 1 (Theorem 1 in Kruse and Popier (2016b)) Under Condition (A) there exists a process $(Y, Z, M)$ such that

- $\quad(Y, Z, M)$ belongs to $\mathbb{S}^{\ell}(0, t)$ for any $t<T$.

- A.s. for any $t \in[0, T], Y_{t} \geq 0$ and $Y$ satisfies Inequality (9).

- The dynamics (11) is verified: for all $0 \leq s \leq t<T$ :

$$
Y(s)=Y(t)+\int_{s}^{t} f(t, Y(r), Z(r)) d r-\int_{s}^{t} Z(r) d W(r)+M(t)-M(s) .
$$

- If the filtration $\mathbb{F}$ is left-continuous at time T, (5) holds a.s.

$$
\liminf _{t \rightarrow T} Y(t) \geq \xi=Y(T) .
$$

Any process $(\tilde{Y}, \tilde{Z}, \tilde{M})$ satisfying the previous four items is called a supersolution of the BSDE (1) with singular terminal condition $\xi$.

The solution $(Y, Z, M)$ obtained by approximation is minimal, that is, if $(\tilde{Y}, \widetilde{Z}, \tilde{M})$ is another nonnegative supersolution, then $\mathbb{P}$-a.s. for all $t \in[0, T], \widetilde{Y}(t) \geq Y(t)$.

Remarks 3 In Kruse and Popier (2016b), we suppose that the filtration $\mathbb{F}$ is quasi left-continuous, which implies that it is left-continuous at time T. This stronger condition is not used to construct the solution if $\xi \in L^{p}(\Omega)$ and the minimal supersolution in the singular case (see Bouchard et al. (2018) for more details on this technical point).

Note that on $Z$ we also have a stronger integrability result:

Proposition 2 (Proposition 4.3 in Popier (2016)) Under Condition (A), there exists a constant $C$ independent of $n$ such that the process $Z^{n}$ satisfies:

$$
\mathbb{E}\left\{\left[\int_{0}^{T}(T-s)^{\rho}\left(\left|Z^{n}(s)\right|^{2}\right) d s\right]^{\ell / 2}\right\} \leq C .
$$


The constant $\rho$ satisfies $\rho>\frac{2}{q}+2\left(1-\frac{1}{\ell}\right)=\frac{2}{q}+\frac{2}{\ell^{*}}$, if $\ell^{*}$ is the Hölder conjugate of $\ell$.

In the sequel, we assume that:

(H) The constant $q$ in Hypothesis (A5) verifies that $q>2$. The filtration $\mathbb{F}$ is left-continuous at time $T$.

Without loss of generality, we assume that $1<\ell<\frac{2 q}{2+q}$ in Condition (A6) such that $\frac{2}{q}+2\left(1-\frac{1}{\ell}\right)<1$ and we choose $\rho<1$ in the above proposition. In particular, if the generator is $f(y)=-y|y|^{q}$ (Example 1), then we work with $q>2$ and $\ell=1$, which was supposed in Popier (2006). In this particular case, the constant $C$ of Proposition 2 is explicitly given by: $C=16(1 / q)^{2 / q}$.

\subsection{Functional Itô calculus}

We adopt the notations and the setting developed in Cont (2016); Cont and Fournié (2013). We simply copy the main definitions; all details are found in these two works.

Consider $D\left([0, T], \mathbb{R}^{d} \times S_{d}^{+}\right)$, the space of all càdlàg functions defined on $[0, T]$ with values in $\mathbb{R}^{d} \times S_{d}^{+}$, where $S_{d}^{+}$is the set of positive $d \times d$ matrices. For a path $\omega \in D\left([0, T], \mathbb{R}^{d} \times S_{d}^{+}\right), \omega_{t}=\omega(t \wedge \cdot)$ is the path stopped at time $t$ and $\omega_{t-}=$ $\omega \mathbf{1}_{[0, t[}+\omega(t-) \mathbf{1}_{[t, T]}$, where $\omega(t-)$ is the left limit of $\omega$ at time $t$.

The space of stopped paths $\Upsilon$ is defined as the quotient space of $[0, T] \times$ $D\left([0, T], \mathbb{R}^{d} \times S_{d}^{+}\right)$by the equivalence relation:

$$
(t, \omega) \sim\left(t^{\prime}, \omega^{\prime}\right) \Leftrightarrow\left(t=t^{\prime} \text { and } \omega_{t}=\omega^{\prime} t^{\prime}\right) .
$$

This space is endowed with the distance

$$
d_{\infty}\left((t, \omega),\left(t^{\prime}, \omega^{\prime}\right)\right)=\left\|\omega_{t}-\omega^{\prime}{ }_{t^{\prime}}\right\|_{\infty}+\left|t-t^{\prime}\right|
$$

And $\left(\Upsilon, d_{\infty}\right)$ is a metric space and a closed subset of $\left([0, T] \times D\left([0, T], \mathbb{R}^{d} \times\right.\right.$ $\left.\left.S_{d}^{+}\right) ;\|\cdot\|_{\infty}\right)$. The notion of non-anticipative, continuous, left-continuous, and boundedness-preserving functionals is defined in (Cont and Fournié 2013, Definitions $2.1,2.3,2.4$, and 2.5$)$, and we denote by $\mathbb{C}^{0,0}\left([0, T)\right.$ ) (resp., $\mathbb{C}^{0,0}([0, T)$ ), resp., $\mathbb{B}([0, T))$ ) the set of continuous (resp., left-continuous, resp., boundednesspreserving) functions on $\Upsilon$. Let $\omega=(x, v)$ with $x \in D\left([0, T], \mathbb{R}^{d}\right)$ and $v \in$ $D\left([0, T], S_{d}^{+}\right)$. We identify $D\left([0, T], \mathbb{R}^{d} \times S_{d}^{+}\right)$and $D\left([0, T], \mathbb{R}^{d}\right) \times D\left([0, T], S_{d}^{+}\right)$. In the sequel, we assume that $F: \Upsilon \rightarrow \mathbb{R}$ is a non-anticipative functional with predictable dependence with respect to $v$ :

$$
\forall(t, \omega=(x, v)) \in \Upsilon, \quad F(t, x, v)=F\left(t, x_{t}, v_{t-}\right) .
$$

Let us briefly recall the definition of the horizontal and vertical derivatives. Let $F: \Upsilon \rightarrow \mathbb{R}$ be a non-anticipative functional. The horizontal derivative $\mathcal{D} F$ of $F$ at 
$(x, v) \in D\left([0, t], \mathbb{R}^{d} \times S_{d}^{+}\right)$is the limit (if it exists)

$$
\mathcal{D} F(t, x, v)=\lim _{h \downarrow 0} \frac{F\left(t+h, x_{t}, v_{t}\right)-F\left(t, x_{t}, v_{t}\right)}{h} .
$$

If the limit exists for all $(x, v)$, the map $\mathcal{D}: \Upsilon \rightarrow \mathbb{R}$ defines a nonanticipative functional $\mathcal{D} F$, called the horizontal derivative (see (Cont and Fournié 2013, Definition 3.1)).

From Cont and Fournié (2013, Definition 3.2) $F$ is vertically differentiable at $(t, x, v)$ if the map defined on $\mathbb{R}^{d}$ by $e \mapsto F\left(t, x_{t}+e \mathbf{1}_{[t, T]}, v_{t}\right)$ is differentiable at 0 . The vertical derivative $\nabla_{\omega} F$ at $(t, x, v)$ is the gradient of the previous map:

$$
\nabla_{\omega} F(t, x, v)=\left(\lim _{h \rightarrow 0} \frac{F\left(t, x_{t}+h e_{i} \mathbf{1}_{[t, T]}, v_{t}\right)-F\left(t, x_{t}, v_{t}\right)}{h}, i=1, \ldots, d\right),
$$

where $\left(e_{i}, i=1, \ldots, d\right)$ is the canonical basis in $\mathbb{R}^{d}$. Let us recall (Cont and Fournié 2013, Definition 3.6):

Definition $2\left(\mathbb{C}^{1, k}\right.$ functionals) Define $\mathbb{C}^{1, k}([0, T))$ as the set of left-continuous functionals $F \in \mathbb{C}_{l}^{0,0}$ such that

- $F$ admits a horizontal derivative $\mathcal{D} F(t, \omega)$ for all $(t, \omega) \in \Upsilon$, and the map $\mathcal{D} F(t, \cdot):\left(D\left([0, T], \mathbb{R}^{d} \times S_{d}^{+}\right),\|.\|_{\infty}\right) \rightarrow \mathbb{R}$ is continuous for each $t \in[0, T[;$

- $F$ is $k$ times vertically differentiable with $\nabla_{\omega}^{j} F \in \mathbb{C}_{l}^{0,0}$.

We define $\mathbb{C}_{b}^{1, k}([0, T))$ as the set of functionals $F \in \mathbb{C}^{1, k}$ such that $\mathcal{D} F$, $\nabla_{\omega} F, \ldots, \nabla_{\omega}^{k} F$ belong to $\mathbb{B}(\Upsilon)$.

In the sequel, we will use the change of variable formulas.

Theorem 4.1 in (Cont and Fournié 2013). Let $F \in \mathbb{C}_{b}^{1,2}$ verifying (12). Let $X$ be a continuous $\mathbb{R}^{d}$-valued semimartingale with absolutely continuous quadratic variation

$$
[X](t)=\int_{0}^{t} A(u) d u,
$$

where $A$ is an $S_{d}^{+}$-valued process. Then, for $t \in[0, T]$

$$
\begin{aligned}
F\left(t, X_{t}, A_{t}\right)= & F\left(0, X_{0}, A_{0}\right)+\int_{0}^{t} \mathcal{D} F\left(u, X_{u}, A_{u}\right) d u \\
& +\int_{0}^{t} \nabla_{\omega} F\left(u, X_{u}, A_{u}\right) d X(u)+\frac{1}{2} \int_{0}^{t} \operatorname{Tr}\left(\nabla_{\omega}^{2} F\left(u, X_{u}, A_{u}\right) d[X](u)\right),
\end{aligned}
$$

where $X(t)$ denotes the value at time $t$, while $X_{t}=(X(u), u \in[0, t])$ the path up to time $t$.

This result implies, in particular, that $\mathcal{X}=F(\cdot, X, A)$ is a continuous semimartingale for any $F \in \mathbb{C}_{b}^{1,2}$.

In the sequel, we need some integrability properties of $\mathcal{X}$. Let us recall that the classical norm on semimartingales is defined in Dellacherie and Meyer (1980), section VII.3 (98.1)-(98.2) or (Protter 2004), section V.2. Nevertheless, this norm is not sufficient in our case and we follow the ideas of (Cont 2016, section 7.5). For 
$p \geq 1$, we define $\mathcal{A}^{p}(\mathbb{F})$ as the set of continuous $\mathbb{F}$-predictable absolutely continuous processes $H=H(0)+\int_{0}^{\cdot} h(t) d t$ with finite variation such that

$$
\|H\|_{\mathcal{A}^{p}}^{p}=\mathbb{E}\left(|H(0)|^{p}+\int_{0}^{T}|h(t)|^{p} d t\right)<+\infty .
$$

We consider the direct sum

$$
\mathcal{S}^{p}=\mathbb{M}^{p}(0, T) \oplus \mathcal{A}^{p}(\mathbb{F})
$$

Any process $S \in \mathcal{S}^{p}$ is an $\mathbb{F}$-adapted special semimartingale with a unique decomposition $S=M+H$, where $M \in \mathbb{M}^{p}(0, T)$ with $M(0)=0$ and $H \in \mathcal{A}^{p}(\mathbb{F})$ with $H(0)=0$. Let us remark that by Jensen's inequality, the norm defined on $\mathcal{S}^{p}$ is stronger than the norm of semimartingales defined in Dellacherie and Meyer (1980). Moreover, if $S \in \mathcal{S}^{p}$, then $S \in \mathbb{D}^{p}(0, T)$ by the Burkholder-Davis-Gundy inequality. The interested reader can find in (Cont 2016, Chapter 7) how the vertical and horizontal derivatives are defined on this space $\mathcal{S}^{p}$.

\section{Obtaining (6) in the non-Markovian setting}

First, note that we do not impose any further condition on the generator. In the sequel, we assume that $X$ is the solution of the SDE:

$$
X(t)=\zeta(t)+\int_{0}^{t} b\left(s, X_{s}\right) \mathrm{d} s+\int_{0}^{t} \sigma\left(s, X_{S}\right) \mathrm{d} W(s)
$$

The coefficients $b(\cdot, \cdot, \phi): \Omega \times[0, T] \rightarrow \mathbb{R}^{d}$ and $\sigma(\cdot, \cdot, \phi): \Omega \times[0, T] \rightarrow \mathbb{R}^{d \times d}$ are progressively measurable stochastic processes for every $\phi \in C\left([0, T] ; \mathbb{R}^{d}\right)$ that satisfy the standard conditions:

- $\quad b, \sigma$ are Lipschitz continuous w.r.t. $\phi$ uniformly in $t$ and $\omega$, i.e., there exists a constant $K_{b, \sigma}$ such that for any $(\omega, t) \in \Omega \times[0, T]$, for any $\phi$ and $\psi$ in $C\left([0, T] ; \mathbb{R}^{d}\right)$ : a.s.

$$
|b(t, \phi)-b(t, \psi)|+|\sigma(t, \phi)-\sigma(t, \psi)| \leq K_{b, \sigma}\left\|\phi_{t}-\psi_{t}\right\|_{\infty} .
$$

- $\quad b$ and $\sigma$ grow at most linearly:

$$
|b(t, 0)|+|\sigma(t, 0)| \leq C_{b, \sigma} .
$$

- $\zeta$ is a progressively measurable continuous stochastic process such that for some $\varrho>0, \zeta \in \mathbb{D}^{\varrho}(0, T)$.

Recall that $\phi_{t}$ is the stopped path of $\phi$, which implies that

$$
\left\|\phi_{t}-\psi_{t}\right\|_{\infty}=\sup \{|\phi(u)-\psi(u)|, 0 \leq u \leq t\} .
$$

Let us emphasize that $X$ is not a Markovian process since the drift and the volatility matrix may depend on the whole trajectory of $X$. Under those assumptions, the 
forward SDE (14) has a unique strong continuous solution $X$ (see (Pardoux and Rascanu 2014, Theorem 3.17)), such that

$$
\mathbb{E}\left[\sup _{t \in[0, T]}|X(t)|^{\varrho}\right] \leq C_{\varrho} .
$$

To simplify the notation, the dimensions of $X$ and of the Brownian motion are the same. But this condition is not crucial and we can also work with different dimensions. The process $X$ is a continuous semimartingale with

$$
[X](t)=\int_{0}^{t} \sigma\left(s, X_{s}\right) \sigma^{*}\left(s, X_{s}\right) d s=\int_{0}^{t} A(s) d s .
$$

We assume that Condition $(\mathbf{C})$ holds, namely:

(C1) There exists a measurable function $\Phi: \mathbb{R} \rightarrow[0,+\infty]$ and $F \in \mathbb{C}_{b}^{1,2}$ such that

$$
\xi=\Phi\left(F\left(T, X_{T}, A_{T}\right)\right) .
$$

We denote $\mathcal{R}=\{\Phi<+\infty\}$ which is supposed to be an open subset of $\mathbb{R}$ and we suppose that $\mathbb{P}(\xi=\infty)>0$. The singular set $\mathfrak{S}_{\xi}$ is the inverse image of $\{\Phi=+\infty\}$ by $F\left(T, X_{T}, A_{T}\right)$.

(C2) For any compact set $\mathcal{K} \subset \mathcal{R}, \mathbb{E}\left(\xi \mathbf{1}_{\mathcal{K}}\left(F\left(T, X_{T}, A_{T}\right)\right)\right)<+\infty$.

(C3) $F(\cdot, X, A)$ is in $\mathcal{S}^{p}$ for $p=\frac{q+1}{q} \ell^{*}$, where $\ell^{*}$ is the Hölder conjugate of the constant $\ell>1$ of Condition (A6).

(C4) $\nabla_{\omega} F$ and $\zeta$ are in $\mathbb{D}^{\ell^{*}}(0, T)$.

The Itô formula (13), together with (14), implies that $F\left(t, X_{t}, A_{t}\right)=F\left(0, X_{0}, A_{0}\right)+\int_{0}^{t} \Theta_{1}(u) d u+\int_{0}^{t} \nabla_{\omega} F\left(u, X_{u}, A_{u}\right) \sigma\left(u, X_{u}\right) d W(u)$, with

$$
\begin{aligned}
\Theta_{1}(s)=\left\{\mathcal{D} F\left(s, X_{s}, A_{s}\right)+\nabla_{\omega} F\right. & \left(s, X_{s}, A_{s}\right) b\left(s, X_{s}\right) \\
& \left.+\frac{1}{2} \operatorname{Tr}\left(\nabla_{\omega}^{2} F\left(s, X_{s}, A_{s}\right) A(s)\right)\right\} .
\end{aligned}
$$

From (C3), we obtain that

$$
\mathbb{E} \int_{0}^{T}\left|\Theta_{1}(s)\right|^{p} d s<+\infty .
$$

Moreover, (C3) implies that $F(\cdot, X, A)$ is in $\mathbb{D}^{p}(0, T)$

$$
\mathbb{E}\left[\sup _{t \in[0, T]}\left|F\left(t, X_{t}, A_{t}\right)\right|^{p}\right]<+\infty,
$$

and that $\sigma(\cdot, X) \nabla_{\omega} F(\cdot, X, A)$ belongs to $\mathbb{H}^{p}(0, T)$

$$
\mathbb{E}\left[\left(\int_{0}^{T}\left[\left(\nabla_{\omega} F\left(s, X_{s}, A_{s}\right)\right)^{*} A(s) \nabla_{\omega} F\left(s, X_{s}, A_{s}\right)\right] \mathrm{d} s\right)^{p / 2}\right]<+\infty .
$$


Remarks 4 Recall that since $q>2$, in order to apply Proposition 2, we have chosen $\ell<\frac{2 q}{q+2}$. Hence,

$$
p=\frac{q+1}{q} \ell^{*}>2 \frac{q+1}{q-2}=2+\frac{6}{q-2} .
$$

In particular, if $q$ is close to $2, p$ is large.

Instead of Condition 3, we could assume that $\nabla_{\omega} F$ is also in $\mathcal{S}^{p}$, following the idea of (Cont 2016, section 7.5).

Let us state our main result.

Theorem 1 Under the hypotheses (A1)-(A6), (H), and $(\mathrm{C} 1)-(\mathrm{C} 4)$, the minimal supersolution Y satisfies a.s.

$$
\liminf _{t \rightarrow T} Y_{t}=\xi
$$

\subsection{Proof of Theorem 1}

Let us explain the key point of the proof, before going into details. From Proposition 1, the minimal supersolution $(Y, Z, M)$ satisfies (5): a.s.

$$
\liminf _{t \rightarrow T} Y(t) \geq \xi=Y(T) .
$$

The goal is to show that the inequality is in fact an equality. On the singular set $\mathfrak{S}_{\xi}$, we deduce that

$$
\liminf _{t \rightarrow T} Y(t) \geq+\infty=\xi .
$$

Hence, we prove the result on the complement of the singular set. Assume that

$$
\lim _{t \rightarrow T} \mathbb{E}\left[Y_{t} \phi\left(F\left(t, X_{t}, A_{t}\right)\right)\right]=\mathbb{E}\left[\xi \phi\left(F\left(T, X_{T}, A_{T}\right)\right)\right]
$$

for the following class of test functions $\phi$ :

$$
\mathfrak{C}=\left\{\phi=\psi^{\gamma}: \quad \psi \in C_{b}^{\infty}\left(\mathbb{R} ; \mathbb{R}_{+}\right) \text {with } \operatorname{supp}(\psi) \subset \mathcal{R} \text { and } \gamma>\frac{2(q+1)}{q}\right\} .
$$

With the continuity of $t \mapsto \phi\left(F\left(t, X_{t}, A_{t}\right)\right)$ and Fatou's lemma, (18) yields to

$$
\begin{aligned}
\mathbb{E}\left[\left(\liminf _{t \rightarrow T} Y_{t}\right) \phi\left(F\left(T, X_{T}, A_{T}\right)\right)\right] & \leq \lim _{t \rightarrow T} \mathbb{E}\left[Y_{t} \phi\left(F\left(t, X_{t}, A_{t}\right)\right)\right] \\
& =\mathbb{E}\left[\xi \phi\left(F\left(T, X_{T}, A_{T}\right)\right)\right] .
\end{aligned}
$$

Since (5) holds, it follows that a.s. on $\{\xi<+\infty\}$ :

$$
\liminf _{t \rightarrow T} Y(t)=\xi=Y(T) .
$$

The proof of (18) is divided into several steps:

- We use functional Itô formula, not with $Y$ because of the terminal singularity, but with the approximation $Y^{n}$. 
- We show that all terms in the Itô decomposition are uniformly bounded (in mean) w.r.t. $n$.

- These uniform estimates are sufficient to pass to the limit on $n$.

- Finally, we derive (18).

Let $\left(Y^{n}, Z^{n}, M^{n}\right)$ be the solution of the BSDE (7) with terminal condition $\xi \wedge n$ and generator $f_{n}$ given by (8):

$$
Y^{n}(t)=\xi \wedge n+\int_{t}^{T} f_{n}\left(s, Y^{n}(s), Z^{n}(s)\right) \mathrm{d} s-\int_{t}^{T} Z^{n}(s) \mathrm{d} W(s)-M^{n}(T)+M^{n}(t)
$$

Let us emphasize that the process $Y^{n}$ is bounded on $\Omega \times[0, T]$. Indeed, since the terminal condition and the quantity $f_{n}(t, 0,0)$ are nonnegative and bounded by $n$, the comparison principle for BSDEs (see Kruse and Popier (2016a)) states that a.s. for any $t \in[0, T]: 0 \leq Y^{n}(t) \leq n(1+T)$. Let $\phi: \mathbb{R} \rightarrow \mathbb{R}_{+}$be a $C^{\infty}$-function with compact support included in $\mathcal{R}$.

Step 1 . We apply Itô's formula to the process $Y^{n} \phi(F(\cdot, X, A))$ between 0 and $t$ :

$$
\begin{aligned}
Y^{n}(t) \phi\left(F\left(t, X_{t}, A_{t}\right)\right)= & Y^{n}(0) \phi\left(F\left(0, X_{0}, A_{0}\right)\right)+\int_{0}^{t} \phi\left(F\left(s, X_{s}, A_{s}\right)\right) Z^{n}(s) d W(s) \\
& +\int_{0}^{t} Y^{n}(s) \phi^{\prime}\left(F\left(s, X_{s}, A_{s}\right)\right) \nabla_{\omega} F\left(s, X_{s}, A_{s}\right) \sigma\left(s, X_{s}\right) d W(s) \\
& +\int_{0}^{t} \phi\left(F\left(s, X_{s}, A_{s}\right)\right) d M^{n}(s) \\
& +\int_{0}^{t} \phi^{\prime}\left(F\left(s, X_{s}, A_{s}\right)\right) \nabla_{\omega} F\left(s, X_{s}, A_{s}\right) \sigma\left(s, X_{s}\right) Z^{n}(s) d s \\
& -\int_{0}^{t} f_{n}\left(s, Y^{n}(s), Z^{n}(s)\right) \phi\left(F\left(s, X_{s}, A_{s}\right)\right) d s \\
& +\int_{0}^{t} Y^{n}(s) \phi^{\prime}\left(F\left(s, X_{s}, A_{s}\right)\right) \Theta_{1}(s) d s \\
& +\frac{1}{2} \int_{0}^{t} Y^{n}(s) \phi^{\prime \prime}\left(F\left(s, X_{s}, A_{s}\right)\right) \Theta_{2}(s) d s
\end{aligned}
$$

where $\Theta_{1}$ is given by (16) and with

$$
\Theta_{2}(s)=\frac{1}{2}\left(\nabla_{\omega} F\left(s, X_{s}, A_{s}\right) \sigma\left(s, X_{s}\right)\right)\left(\nabla_{\omega} F\left(s, X_{s}, A_{s}\right) \sigma\left(s, X_{s}\right)\right)^{*} .
$$

Now, we decompose the quantity with the generator $f_{n}$ as follows: 


$$
\begin{aligned}
\int_{0}^{t} \phi & \left(F\left(s, X_{s}, A_{s}\right)\right) f_{n}\left(s, Y^{n}(s), Z^{n}(s)\right) d s \\
= & \int_{0}^{t} \phi\left(F\left(s, X_{s}, A_{s}\right)\right)\left(f\left(s, Y^{n}(s), 0\right)-f^{0}(s)\right) d s \\
& +\int_{0}^{t} \phi\left(F\left(s, X_{s}, A_{s}\right)\right)\left(f^{0}(s) \wedge n\right) d s \\
& +\int_{0}^{t} \phi\left(F\left(s, X_{s}, A_{s}\right)\right)\left(f\left(s, Y^{n}(s), Z^{n}(s)\right)-f\left(s, Y^{n}(s), 0\right)\right) d s \\
= & \int_{0}^{t} \phi\left(F\left(s, X_{s}, A_{s}\right)\right)\left(f\left(s, Y^{n}(s), 0\right)-f^{0}(s)\right) d s \\
& +\int_{0}^{t} \phi\left(F\left(s, X_{s}, A_{s}\right)\right)\left(f^{0}(s) \wedge n\right) d s \\
& +\int_{0}^{t} \phi\left(F\left(s, X_{s}, A_{s}\right)\right) \zeta^{n}(s) Z^{n}(s) d s
\end{aligned}
$$

where $\zeta_{s}^{n}$ is a $d$-dimensional random vector defined by: for $i=1, \ldots, d$

$$
\zeta^{i, n}(s)=\frac{\left(f\left(s, Y^{n}(s), Z^{n}(s)\right)-f\left(s, Y^{n}(s), 0\right)\right)}{Z^{i, n}(s)} \mathbf{1}_{Z^{i, n}(s) \neq 0} .
$$

From Condition (A4), $\left|\zeta^{n}(s)\right| \leq L$. Hence, we obtain

$$
\begin{aligned}
Y^{n}(t) \phi\left(F\left(t, X_{t}, A_{t}\right)\right)= & Y^{n}(0) \phi\left(F\left(0, X_{0}, A_{0}\right)\right) \\
& +\int_{0}^{t} \phi\left(F\left(s, X_{s}, A_{s}\right)\right)\left[Z^{n}(s) d W(s)+d M^{n}(s)\right] \\
& +\int_{0}^{t} Y^{n}(s) \phi^{\prime}\left(F\left(s, X_{s}, A_{s}\right)\right) \nabla_{\omega} F\left(s, X_{s}, A_{s}\right) \sigma\left(s, X_{s}\right) d W(s) \\
& +\int_{0}^{t} \Psi^{n}(s) Z^{n}(s) d s-\int_{0}^{t} \phi\left(F\left(s, X_{s}, A_{s}\right)\right)\left(f^{0}(s) \wedge n\right) d s \\
& -\int_{0}^{t} \phi\left(F\left(s, X_{s}, A_{s}\right)\right)\left(f\left(s, Y^{n}(s), 0\right)-f^{0}(s)\right) d s \\
& +\int_{0}^{t} Y^{n}(s)\left[\phi^{\prime}\left(F\left(s, X_{s}, A_{s}\right)\right) \Theta_{1}(s)\right. \\
& \left.+\phi^{\prime \prime}\left(F\left(s, X_{s}, A_{s}\right)\right) \Theta_{2}(s)\right] d s
\end{aligned}
$$

with

$$
\Psi^{n}(s)=\phi^{\prime}\left(F\left(s, X_{s}, A_{s}\right)\right) \nabla_{\omega} F\left(s, X_{s}, A_{s}\right) \sigma(s, X(s))-\phi\left(F\left(s, X_{s}, A_{s}\right)\right) \zeta^{n}(s)
$$


Recall that for a fixed $n, Y^{n}$ is bounded, $\left(Z^{n}, M^{n}\right)$ belong to $\mathbb{H}^{\kappa}(0, T) \times \mathbb{M}^{\kappa}(0, T)$ for any $\kappa \geq 1$. From Condition 3 on $F(\cdot, X, A)$, taking the expectation in (19) leads to

$$
\begin{aligned}
\mathbb{E}\left[Y^{n}(T) \phi\left(F\left(T, X_{T}, A_{T}\right)\right)\right]= & \mathbb{E}\left[Y^{n}(t) \phi\left(F\left(t, X_{t}, A_{t}\right)\right)\right] \\
& -\mathbb{E}\left[\int_{t}^{T} \phi\left(F\left(s, X_{s}, A_{s}\right)\right)\left(f^{0}(s) \wedge n\right) d s\right] \\
& -\mathbb{E}\left[\int_{t}^{T} \phi\left(F\left(s, X_{s}, A_{s}\right)\right)\left(f\left(s, Y^{n}(s), 0\right)-f^{0}(s)\right) d s\right] \\
& +\mathbb{E}\left[\int _ { t } ^ { T } Y ^ { n } ( s ) \left[\phi^{\prime}\left(F\left(s, X_{s}, A_{s}\right)\right) \Theta_{1}(s)\right.\right. \\
& \left.\left.+\phi^{\prime \prime}\left(F\left(s, X_{s}, A_{s}\right)\right) \Theta_{2}(s)\right] d s\right] \\
& +\mathbb{E}\left[\int_{t}^{T} \Psi^{n}(s) Z^{n}(s) d s\right],
\end{aligned}
$$

for any $t \in[0, T]$.

Step 2 . We prove that all terms in (20) are uniformly bounded. From the assumptions (C1) and (C2) on $\xi=\Phi\left(F\left(T, X_{T}, A_{T}\right)\right)$, since $Y^{n}(T)=\xi \wedge n$, there exists a constant $C_{1}$, given by:

$$
C_{1}=\mathbb{E}\left[\Phi\left(F\left(T, X_{T}, A_{T}\right)\right) \phi\left(F\left(T, X_{T}, A_{T}\right)\right)\right]<+\infty,
$$

such that:

$$
\sup _{n \in \mathbb{N}} \mathbb{E}\left[Y^{n}(T) \phi\left(F\left(T, X_{T}, A_{T}\right)\right] \leq C_{1}\right.
$$

Since $Y^{n}(t) \leq Y(t)$ for all $n \in \mathbb{N}$ and any $t<T$, from the a priori estimate (9), Assumption (A6), and the boundedness of $\phi$, we have

$$
\mathbb{E}\left(Y^{n}(t) \phi\left(F\left(t, X_{t}, A_{t}\right)\right)\right) \leq \frac{K_{\ell, L}}{(T-t)^{1 / q+1}} \mathbb{E}\left(\Gamma(t) \phi\left(F\left(t, X_{t}, A_{t}\right)\right)\right)<+\infty
$$

In particular, we deduce the existence of a constant $C_{2}$ such that

$$
\sup _{n \in \mathbb{N}} \mathbb{E}\left[Y^{n}(0) \phi\left(F\left(0, X_{0}, A_{0}\right)\right] \leq C_{2}\right.
$$

Since $\phi$ is bounded and $f^{0} \in L^{1}((0, T) \times \Omega)$ (Condition (A6)), we deduce that there exists a constant $C_{3}$ such that:

$$
\sup _{n \in \mathbb{N}} \mathbb{E}\left[\int_{0}^{T} \phi\left(F\left(s, X_{s}, A_{s}\right)\right)\left(f_{s}^{0} \wedge n\right) d s\right] \leq C_{3}
$$


We use Hölder's and Young's inequalities to obtain:

$$
\begin{aligned}
\int_{0}^{t} \mid & {\left[\phi^{\prime}\left(F\left(s, X_{s}, A_{s}\right)\right) \nabla_{\omega} F\left(s, X_{s}, A_{s}\right) \sigma\left(s, X_{s}\right)+\phi\left(F\left(s, X_{s}, A_{s}\right)\right) \zeta^{n}(s)\right] Z^{n}(s) \mid d s } \\
& \leq \int_{0}^{t}\left|\Psi^{n}(s) Z^{n}(s)\right| d s \\
& \leq\left[\int_{0}^{t}(T-s)^{\rho}\left|Z^{n}(s)\right|^{2} d s\right]^{1 / 2}\left[\int_{0}^{t} \frac{\left|\Psi^{n}(s)\right|^{2}}{(T-s)^{\rho}} d s\right]^{1 / 2} \\
& \leq \frac{1}{\ell}\left[\int_{0}^{t}(T-s)^{\rho}\left|Z^{n}(s)\right|^{2} d s\right]^{\frac{\ell}{2}}+\frac{1}{\ell^{*}}\left[\int_{0}^{t} \frac{\left|\Psi^{n}(s)\right|^{2}}{(T-s)^{\rho}} d s\right]^{\frac{\ell^{*}}{2}} \\
& \leq \frac{1}{\ell}\left[\int_{0}^{t}(T-s)^{\rho}\left|Z^{n}(s)\right|^{2} d s\right]^{\frac{\ell}{2}}+\frac{1}{\ell^{*}}\left(\frac{T^{1-\rho}}{1-\rho}\right)^{\frac{\ell^{*}}{2}} \sup _{t \in[0, T]}\left|\Psi^{n}(t)\right|^{\ell^{*}},
\end{aligned}
$$

where $\rho$ is the constant introduced in Proposition 2. Consequently, taking the expectation, the first term on the right side is bounded. For the second term, $\phi, \phi^{\prime}$, and $\zeta^{n}$ are bounded. From Condition 3, we deduce that there exists a constant $C_{4}$ such that:

$$
\sup _{n \in \mathbb{N}} \mathbb{E} \int_{0}^{T}\left|\Psi_{s}^{n} Z_{s}^{n}\right| d s \leq C_{4} .
$$

Coming back to (20) with $t=0$, and using (21), (23), (24), and (25), we deduce that for any test function $\phi$, there exists a constant $\mathcal{C}=C_{1}+C_{2}+C_{3}+C_{4}$, independent of $n$, such that for any $n$ :

$$
\begin{aligned}
& \mathbb{E} {\left[\int_{0}^{T} Y^{n}(s)\left[\phi^{\prime}\left(F\left(s, X_{s}, A_{s}\right)\right) \Theta_{1}(s)+\phi^{\prime \prime}\left(F\left(s, X_{s}, A_{s}\right)\right) \Theta_{2}(s)\right] d s\right] } \\
&-\mathbb{E}\left[\int_{0}^{T} \phi\left(F\left(s, X_{s}, A_{s}\right)\right)\left(f\left(s, Y^{n}(s), 0\right)-f^{0}(s)\right) d s\right] \\
&= \mathbb{E}\left[Y^{n}(T) \phi\left(F\left(T, X_{T}, A_{T}\right)\right)\right]-\mathbb{E}\left[Y^{n}(0) \phi\left(F\left(0, X_{0}, A_{0}\right)\right)\right] \\
& \quad+\mathbb{E}\left[\int_{0}^{T} \phi\left(F\left(s, X_{s}, A_{s}\right)\right)\left(f^{0}(s) \wedge n\right) d s\right]-\mathbb{E}\left[\int_{0}^{T} \Psi^{n}(s) Z^{n}(s) d s\right] \\
& \leq \mathcal{C} .
\end{aligned}
$$

Now, we treat the two terms in (20) containing $Y^{n}$. With Hölder's inequality, we obtain for $j=1$ or 2

$$
\begin{aligned}
& \int_{0}^{t}\left|Y^{n}(s) \phi^{(j)}\left(F\left(s, X_{s}, A_{s}\right)\right) \Theta_{j}(s)\right| d s \leq\left[\int_{0}^{t} \phi\left(F\left(s, X_{s}, A_{s}\right)\right) a(s)\left|Y^{n}(s)\right|^{1+q} d s\right]^{\frac{1}{q+1}} \\
& \times\left[\int_{0}^{t} a(s)^{-1 / q} \phi\left(F\left(s, X_{s}, A_{s}\right)\right)^{-1 / q}\left|\phi^{(j)}\left(F\left(s, X_{s}, A_{s}\right)\right)\right|^{\frac{q+1}{q}}\left|\Theta_{j}(s)\right|^{\frac{q+1}{q}} d s\right]^{\frac{q}{q+1}} .
\end{aligned}
$$

To control the second quantity, we will be more specific about the test-function $\phi$. We assume that $\phi=\psi^{\gamma}$, where $\psi$ belongs to $C_{b}^{\infty}\left(\mathbb{R} ; \mathbb{R}_{+}\right)$with support in $\mathcal{R}$ and 
$\gamma>2(q+1) / q$. Under this setting, there exists a constant $C$ depending only on $\psi$ and $\gamma$ such that

$$
\left|\phi^{\prime}\right|+\left|\phi^{\prime \prime}\right| \leq C \psi^{\gamma-2}
$$

Thus, for $\gamma>2(q+1) / q$ and $j=1$ or 2

$$
\phi\left(F\left(s, X_{s}, A_{s}\right)\right)^{-1 / q}\left|\phi^{(j)}\left(F\left(s, X_{s}, A_{s}\right)\right)\right|^{(q+1) / q} \leq C \psi\left(F\left(s, X_{s}, A_{s}\right)\right)^{\gamma-2(q+1) / q},
$$

which is bounded. By Condition (A6), $a^{-1 / q}$ is in $\mathbb{L}^{\ell}(\Omega \times[0, T])$. By Assumption (C3), the quantity $\left|\Theta_{j}\right|^{\frac{q+1}{q}}$ is in $\mathbb{L}^{\ell^{*}}(\Omega \times[0, T])$. We deduce that there exists a constant $\widehat{\mathcal{C}}$ such that for any $t \in[0, T]$ and any $n$ :

$$
\begin{aligned}
& \mathbb{E}\left[\int_{0}^{t}\left|Y^{n}(s) \phi^{(j)}\left(F\left(s, X_{s}, A_{s}\right)\right) \Theta_{j}(s)\right| d s\right] \\
& \quad \leq \widehat{\mathcal{C}}\left[\mathbb{E} \int_{0}^{t} a(s) \phi\left(F\left(s, X_{s}, A_{s}\right)\right)\left(Y^{n}(s)\right)^{q+1} d s\right]^{\frac{1}{q+1}} .
\end{aligned}
$$

Now, by Condition (A5), note that:

$$
\begin{gathered}
-\int_{0}^{t} \phi\left(F\left(s, X_{s}, A_{s}\right)\right)\left(f\left(s, Y^{n}(s), 0\right)-f(s, 0,0)\right) d s \\
\geq \int_{0}^{t} \phi\left(F\left(s, X_{s}, A_{s}\right)\right) a(s)\left|Y^{n}(s)\right|^{1+q} d s .
\end{gathered}
$$

If we denote

$$
\mathcal{I}^{n}=\mathbb{E} \int_{0}^{T} \phi\left(F\left(s, X_{s}, A_{s}\right)\right) a(s)\left|Y^{n}(s)\right|^{1+q} d s,
$$

the inequalities (26), (27) and (28) lead to:

$$
\begin{aligned}
\mathcal{I}^{n}-\widehat{\mathcal{C}}\left(\mathcal{I}^{n}\right)^{\frac{1}{q+1}} \leq \mathbb{E}\left[\int_{t}^{T} Y^{n}(s)\left[\phi^{\prime}\left(F\left(s, X_{s}, A_{s}\right)\right) \Theta_{1}(s)+\phi^{\prime \prime}\left(F\left(s, X_{s}, A_{s}\right)\right) \Theta_{2}(s)\right] d s\right] \\
\quad-\mathbb{E}\left[\int_{t}^{T} \phi\left(F\left(s, X_{s}, A_{s}\right)\right)\left(f\left(s, Y^{n}(s), 0\right)-f^{0}(s)\right) d s\right] \\
\leq \mathcal{C} .
\end{aligned}
$$

Thereby, we deduce the existence of a constant $C_{5}$ such that for any $n$ :

$$
0 \leq \mathcal{I}^{n}=\mathbb{E} \int_{0}^{T} a(s) \phi\left(F\left(s, X_{s}, A_{s}\right)\right)\left|Y^{n}(s)\right|^{1+q} d s \leq C_{5}<+\infty .
$$

Step 3. We prove that we can pass to the limit on $n$ in (20). By the monotone convergence theorem, we can pass to the limit when $n$ goes to $+\infty$ in the first three terms of (20). If the constant $\chi$ in (A2) is nonpositive, then $y \mapsto f(t, y, 0)-f^{0}(s)$ 
is nonincreasing and we can also apply the monotone convergence theorem to the fourth term of (20). If not, substracting $\chi Y^{n}$ from $f\left(s, Y^{n}(s), 0\right)$, we have:

$$
\begin{aligned}
\mathbb{E} & {\left[\int_{t}^{T} \phi\left(F\left(s, X_{s}, A_{s}\right)\right)\left(f\left(s, Y^{n}(s), 0\right)-f^{0}(s)\right) d s\right] } \\
= & \mathbb{E}\left[\int_{t}^{T} \phi\left(F\left(s, X_{s}, A_{s}\right)\right)\left(f\left(s, Y^{n}(s), 0\right)-f^{0}(s)-\chi Y^{n}(s)\right) d s\right] \\
& +\chi \mathbb{E}\left[\int_{t}^{T} \phi\left(F\left(s, X_{s}, A_{s}\right)\right) Y^{n}(s) d s\right] .
\end{aligned}
$$

Now from (A2), the mapping $y \mapsto f(t, y, 0)-f^{0}(s)-\chi y$ is nonincreasing and we can also apply the monotone convergence theorem. Furthermore, using the same arguments as for the estimate (27) yields to the existence of a constant $C$ such that for all $n \in \mathbb{N}$ :

$\mathbb{E}\left[\int_{t}^{T} \phi\left(F\left(s, X_{s}, A_{s}\right)\right) Y^{n}(s) d s\right] \leq C\left[\mathbb{E} \int_{0}^{t} a(s) \phi\left(F\left(s, X_{s}, A_{s}\right)\right)\left(Y^{n}(s)\right)^{q+1} d s\right]^{\frac{1}{q+1}}$.

For this additional term and the last two terms of (20) (containing $Y^{n}$ and $Z^{n}$ ), we summarize the arguments (see details in Popier (2006)). Estimate (29) shows that the sequence $a^{\frac{1}{1+q}} \phi(F(\cdot, X, A))^{\frac{1}{1+q}} Y^{n}$ is bounded in $\mathbb{L}^{1+q}(\Omega \times(0, T))$. Using a weak convergence result and extracting a subsequence if necessary, and arguing as in the proof of Estimate (27), we pass to the limit in the term

$$
\mathbb{E}\left[\int_{t}^{T} Y^{n}(s)\left[\phi^{\prime}\left(F\left(s, X_{s}, A_{s}\right)\right) \Theta_{1}(s)+\phi^{\prime \prime}\left(F\left(s, X_{s}, A_{s}\right)\right) \Theta_{2}(s)\right] d s\right] .
$$

From Proposition 2, there exists a subsequence, which we denote as $(T-\cdot)^{1 /(2 \rho)} Z^{n}$, and which converges weakly in the space $\mathbb{L}^{2}(\Omega \times(0, T))$ to the limit $(T-\cdot)^{1 /(2 \rho)} Z$, because we know that $Z^{n}$ converges to $Z$ in $\mathbb{H}^{\ell}(\Omega \times(0, T-\delta))$ for all $\delta>0$. Define on $[0, T)$ the $d$-dimensional random vector $\zeta$ by: for $i=1, \ldots, d$

$$
\zeta^{i}(s)=\frac{(f(s, Y(s), Z(s))-f(s, Y(s), 0))}{Z^{i}(s)} \mathbf{1}_{Z^{i}(s) \neq 0}
$$

and $\Psi$ is defined as $\Psi^{n}$, replacing $\zeta^{n}$ with $\zeta$. Again, $|\zeta(s)| \leq K$ and we showed that $\Psi^{n} /(T-\cdot)^{1 /(2 \rho)}$ and $\Psi /(T-\cdot)^{1 /(2 \rho)}$ are in $\mathbb{L}^{\ell^{*}}\left(\Omega ; \mathbb{L}^{2}(0, T)\right)$. For any $\varepsilon>0$, we deduce that there exists $\delta>0$ such that

$$
\mathbb{E} \int_{T-\delta}^{T}\left(\left|\Psi^{n}(s) Z^{n}(s)\right|+|\Psi(s) Z(s)|\right) \mathrm{d} s \leq \varepsilon / 2
$$

On the interval $[0, T-\delta]$, the sequence $\left(Y^{n}, Z^{n}\right)$ converges to $(Y, Z)$ in $\mathbb{D}^{\ell}(0, T-$ $\delta) \times \mathbb{H}^{\ell}(0, T-\delta)$. Hence,

$$
\lim _{n \rightarrow+\infty} \mathbb{E} \int_{0}^{T-\delta}\left|\Psi^{n}(s) Z^{n}(s)-\Psi(s) Z(s)\right| \mathrm{d} s=0 .
$$


In other words, the sequence $\Psi^{n} Z^{n}$ converges in $\mathbb{L}^{1}(\Omega \times(0, T))$ to $\Psi Z$ and

$$
\mathbb{E} \int_{0}^{T}|\Psi(s) Z(s)| d s \leq C_{4} .
$$

Passing to the limit in (20) implies:

$$
\begin{aligned}
\mathbb{E} & {\left[\xi \phi\left(F\left(T, X_{T}, A_{T}\right)\right)\right]=\mathbb{E}\left[Y(t) \phi\left(F\left(t, X_{t}, A_{t}\right)\right)\right] } \\
- & \mathbb{E}\left[\int_{t}^{T} \phi\left(F\left(s, X_{s}, A_{s}\right)\right) f^{0}(s) d s\right] \\
- & \mathbb{E}\left[\int_{t}^{T} \phi\left(F\left(s, X_{s}, A_{s}\right)\right)\left(f(s, Y(s), 0)-f^{0}(s)\right) d s\right] \\
+ & \mathbb{E}\left[\int_{t}^{T} Y(s)\left[\phi^{\prime}\left(F\left(s, X_{s}, A_{s}\right)\right) \Theta_{1}(s)+\phi^{\prime \prime}\left(F\left(s, X_{s}, A_{s}\right)\right) \Theta_{2}(s)\right] d s\right] \\
+ & \mathbb{E}\left[\int_{t}^{T} \Psi(s) Z(s) d s\right] .
\end{aligned}
$$

Step 4. Finally, Estimate (29) also holds with $Y$ (instead of $Y^{n}$ ), and all estimates (24), (25), (27), and (28) are satisfied for the limit processes $Y, Z$, and $\Psi$. Hence, we let $t$ go to $T$ in (30) to get (18):

$$
\lim _{t \rightarrow T} \mathbb{E}\left[Y(t) \phi\left(F\left(t, X_{t}, A_{t}\right)\right)\right]=\mathbb{E}\left[\xi \phi\left(F\left(T, X_{T}, A_{T}\right)\right)\right] .
$$

As explained at the beginning of the proof, this equality is the key point to deduce that a.s.

$$
\liminf _{t \rightarrow T} Y(t)=\xi
$$

This achieves the proof of Theorem 1 .

The proof of Theorem 1 shows that the limit of $Y_{t}$ exists in mean in the following sense: for smooth function $\phi$

$\lim _{t \rightarrow T} \mathbb{E}\left(Y(t) \phi\left(F\left(t, X_{t}, A_{t}\right)\right)\right)= \begin{cases}\mathbb{E}\left(\xi \phi\left(F\left(T, X_{T}, A_{T}\right)\right)\right) & \text { ifsupp }(\phi) \cap \mathfrak{S}_{\xi}=\emptyset, \\ +\infty & \text { if } \mathbb{E}\left(\phi\left(F\left(T, X_{T}, A_{T}\right)\right) \mathbf{1}_{\mathfrak{S}_{\xi}}\right)>0 .\end{cases}$

\subsection{Some examples}

Several examples of smooth functionals are given in Cont (2016) and Cont and Fournié (2013). There are also interesting counterexamples (see (Cont and Fournié 2013, section 3.2)).

First, we can recover the Markovian case if for some smooth function $h \in$ $C^{1,2}\left([0, T] \times \mathbb{R}^{d}\right)$

and if $X$ satisfies the SDE

$$
F\left(t, X_{t}, A_{t}\right)=h(t, X(t)),
$$

$$
X(t)=x+\int_{0}^{t} b(s, X(s)) d s+\int_{0}^{t} \sigma(s, X(s)) d W(s) .
$$

Here, the coefficients $b:[0, T] \times \mathbb{R}^{d} \rightarrow \mathbb{R}^{d}$ and $\sigma:[0, T] \times \mathbb{R}^{d} \rightarrow \mathbb{R}^{d \times d}$ are Lipschitz continuous w.r.t. $x$ uniformly in $t$, and the functions $b$ and $\sigma$ grow 
at most linearly. Under this setting, the SDE has a unique strong continuous solution $X$ such that (15) holds for any $\varrho \geq 1$. Then, $\mathcal{D} F\left(t, X_{t}, A_{t}\right)=\partial_{t} h(t, X(t))$, $\nabla_{\omega} F\left(t, X_{t}, A_{t}\right)=\nabla_{x} h(t, X(t))$ and $\nabla_{\omega}^{2} F\left(t, X_{t}, A_{t}\right)=D_{x}^{2} h(t, X(t))$, where $D_{x}^{2}$ is the Hessian matrix w.r.t. $x$. In this case, Eq. 20 is the same as the classical Itô formula used in Popier (2016). If we assume that $h$ and its derivatives are of linear growth w.r.t. $x$, uniformly in time and $\omega$, then using (15), Assumptions (C3) and (C4) are satisfied.

As a second example, we consider the case where $X$ is the solution of (14) and

$$
F\left(t, X_{t}, A_{t}\right)=\int_{0}^{t} h(s, X(s)) A(s) d s,
$$

where $h$ is a continuous function on $[0, T] \times \mathbb{R}^{d}$. Then, $\mathcal{D} F\left(s, X_{s}, A_{s}\right)=$ $h(s, X(s)) A(s), \nabla_{\omega} F\left(s, X_{s}, A_{s}\right)=0$ and Conditions (C3) and (C4) are satisfied trivially verified if $\varrho$ is sufficiently large and if $h$ is of linear growth w.r.t. $x$. Moreover, Eq. 19 can be simplified:

$$
\begin{aligned}
Y_{t}^{n} \phi\left(F\left(t, X_{t}, A_{t}\right)\right)= & Y_{0}^{n} \phi\left(F\left(0, X_{0}, A_{0}\right)\right) \\
& +\int_{0}^{t} \phi\left(F\left(s, X_{s}, A_{s}\right)\right)\left[Z^{n}(s) d W(s)+d M^{n}(s)\right] \\
& -\int_{0}^{t} f_{n}\left(s, Y_{s}^{n}, Z_{s}^{n}\right) \phi\left(F\left(s, X_{s}, A_{s}\right)\right) d s \\
& +\int_{0}^{t} Y_{s}^{n} \phi^{\prime}\left(F_{s}\left(X_{s}, A_{s}\right)\right) h(s, X(s)) A(s) d s .
\end{aligned}
$$

Other examples are given by (Cont and Fournié 2013, Examples 4 and 5), namely,

$$
F\left(t, x_{t}, v_{t}\right)=x(t)^{2}-\int_{0}^{t} v(u) d u, \quad F\left(t, x_{t}, v_{t}\right)=\exp \left(x(t)-\frac{1}{2} \int_{0}^{t} v(u) d u\right) .
$$

Conditions on $b$ and $\sigma$ are easily found such that (C3) and (C4) hold, especially if $X$ is given by (31).

We now finish with the weak Euler-Maruyama scheme as in Cont and Lu (2016). We still consider the SDE (14) with $b=0$ and the non-anticipative functional $X^{n}$ given by the recursion

$$
X^{n}\left(t_{j+1}\right)=X^{n}\left(t_{j}\right)+\sigma\left(t_{j}, X_{t_{j}}^{n}\right)\left(W\left(t_{j+1}\right)-W\left(t_{j}\right)\right) .
$$

For a Lipschitz functional $g: D\left([0, T], \mathbb{R}^{d}\right) \rightarrow \mathbb{R}$, consider the "weak Euler approximation"

$$
F_{n}(t)=\mathbb{E}\left[g\left(X_{T}^{n}\right) \mid \mathcal{F}_{t}^{W}\right]
$$

of the conditional expectation $\mathbb{E}\left[g\left(X_{T}\right) \mid \mathcal{F}_{t}^{W}\right]$, where $\mathbb{F}^{W}$ is the filtration generated by the Brownian motion $W$. This weak approximation is computed by initializing the scheme on $[0, t]$ with $\omega$ (a path of the Brownian motion) and then iterating the scheme with the increments of the Wiener process between $t$ and $T$. Then, $F_{n} \in \mathbb{C}_{\text {loc }}^{1, \infty}$ (see (Cont and Lu 2016, Theorem 3.1)). Moreover, since we have a martingale, $\Theta_{1}(s)=$ 0. Under our setting and due to (Cont and Lu 2016, Theorem 4.1), (C3) holds. (C4) does not hold on the whole interval $[0, T]$. Nevertheless, this functional is locally 
regular (Cont and Lu 2016, Definition 7) and on our neighbourhood of $T$, one easily gets (C4), provided that $g$ is bounded, for example.

Acknowledgements Not applicable.

\section{Authors' contributions}

Both authors read and approved the final manuscript.

\section{Funding}

Not applicable.

\section{Availability of data and materials}

Not applicable.

\section{Competing interests}

The authors declare that they have no competing interests.

\section{References}

Ankirchner, S., M. Jeanblanc, and T. Kruse. (2014). BSDEs with Singular Terminal Condition and a Control Problem with Constraints, SIAM J. Control Optim. 52, no. 2, 893-913.

Bank, P. and M. Voß. (2018). Linear quadratic stochastic control problems with stochastic terminal constraint, SIAM J. Control Optim. 56, no. 2, 672-699.

Bouchard, B., D. Possamaï, X. Tan, and C. Zhou. (2018). A unified approach to a priori estimates for supersolutions of BSDEs in general filtrations, Ann. Inst. Henri Poincaré, Probab. Stat. 54, no. 1, $154-172$.

Cont, R. (2016). Functional Itô calculus and functional Kolmogorov equations, Birkhäuser/Springer, CRM Barcelona.

Cont, R. and D.-A. Fournié. (2010). A functional extension of the Ito formula, C. R. Math. Acad. Sci. Paris. 348, no. 1-2, 57-61.

Cont, R. and D.-A. Fournié. (2013). Functional Itô calculus and stochastic integral representation of martingales, Ann. Probab. 41, no. 1, 109-133.

Cont, R. and Y. Lu. (2016). Weak approximation of martingale representations, Stoch. Process. Appl. 126, no. 3, 857-882.

Dellacherie, C. and P.-A. Meyer. (1980). Probabilités et potentiel. Théorie des martingales, Chapitres V à VIII, Hermann.

Delong, Ł. (2013). Backward stochastic differential equations with jumps and their actuarial and financial applications, European Actuarial Academy (EAA) Series, Springer, London. BSDEs with jumps.

Dupire, B. (2009). Functional Itô calculus, Bloomberg Portfolio Research Paper No 2009-04FRONTIERS.

Graewe, P., U. Horst, and J. Qiu. (2015). A non-Markovian liquidation problem and backward SPDEs with singular terminal conditions, SIAM J. Control Optim. 53, no. 2, 690-711.

Kruse, T. and A. Popier. (2016). BSDEs with monotone generator driven by Brownian and Poisson noises in a general filtration, Stochastics $\mathbf{8 8}$, no. 4, 491-539.

Kruse, T. and A. Popier. (2016). Minimal supersolutions for BSDEs with singular terminal condition and application to optimal position targeting, Stoch. Process. Appl. 126, no. 9, 2554-2592.

Kruse, T. and A. Popier. (2017). $L^{p}$-solution for BSDEs with jumps in the case $p<2$ : corrections to the paper 'BSDEs with monotone generator driven by Brownian and Poisson noises in a general filtration, Stochastics 89, no. 8, 1201-1227. 
Pardoux, E. and A. Rascanu. (2014). Stochastic Differential Equations, Backward SDEs, Partial Differential Equations, volume 69 of Stochastic Modelling and Applied Probability, Springer-Verlag. https:// doi.org/10.1007/978-3-319-05714-9.

Popier, A. (2006). Backward stochastic differential equations with singular terminal condition, Stoch. Process. Appl 116, no. 12, 2014-2056.

Popier, A. (2016). Limit behaviour of bsde with jumps and with singular terminal condition, ESAIM: PS 20, 480-509.

Protter, P.E. (2004). Stochastic integration and differential equations, volume 21 of Applications of Mathematics (New York), second edition, Springer-Verlag, Berlin. Stochastic Modelling and Applied Probability.

Quenez, M.-C. and A. Sulem. (2013). BSDEs with jumps, optimization and applications to dynamic risk measures, Stoch. Process. Appl. 123, no. 8, 3328-3357.

Sezer, A.D., T. Kruse, and A. Popier. (2019). Backward stochastic differential equations with nonMarkovian singular terminal values, Stoch. Dyn. 19, no. 2, 1950006. 